\title{
DETECTION OF NON-ORTHOGONAL PAM SIGNALS WITH SPECTRAL OVERLAPPLNG
}

\author{
A. M. P. de Lucena ${ }^{(I)}$, J. C. M. Mota ${ }^{(2)}$, and C. C. Cavalcante ${ }^{(2)}$. \\ (1) CRN/CEP, National Institut of Space Research, Fortaleza, CE, Brazil. \\ macilio@roen.inpe.br \\ (2) GTEL/DETI, Federal University of Ceará, Fortaleza, CE, Brazil. \\ \{mota,charles\}@gtel.ufc.br
}

\begin{abstract}
A model for a communication system transmitting two nonotthogonal n-PAM signals with spectral overlapping through an AWGN band-limited channel is established. The system is shown to be equivalent to a discrete time-variant multi-user channel with strong interference among users. A receiver structure based on source separation concepts is proposed. The performance of signal detection in terms of symbol error rate is evaluated using Monte Carlo simulations.
\end{abstract}

\section{INTRODUCTION}

Several communication systems using multiple access strategies, like OFDM [1] and CDMA [2] utilize orthogonal waveform signals to transmit information from different users. The orthogonality among waveforms makes possible spectral superposition of signals that increase the system bandwidth efficiency without power performance degradation.

On the other hand, the modulation system $\{\mathrm{m}-\mathrm{QAM}\}^{2}$ [3] transmits simultaneously two non-orthogonal m-QAM signals with spectral overlapping but with no sacrifice in power budget However, the great limitation of this modulation technique is the channel bandwidth required which must be theoretically infinite to hold the power efficiency of the system.

In this work, we investigate the situation where no orthogonality between waveforms is considered and the channel is band limited.

This paper presents an original mathematical model and analysis of a communication system that transmits at the same time two band-limited n-PAM signals with partial spectral superposition over AWGN channels. Also, a receiver structure is proposed based on source separation concepts.

The rest of the paper is organized as follows. In Section 2, the system is described, expressions for demodulated signals in the proposed mathematical model are shown, and a discrete-time model to the system is presented. In Section 3, symbol separation and detection strategies are proposed. Monte Carlo simulation results comparing the performance of the proposal w.r.t. symbol error rate are presented in Section 4. Finally in Section 5, our conclusions and perspectives are stated.

\section{SYSTEM DESCRIPTION}

The block diagram of system transmitter is given in the Fig.1. The signals $x_{1}(k)$ and $x_{2}(k)$ are the transmitted symbols in the instant $k$ from two independent sources. Symbol duration is $T$, and $f_{1}$ and $f_{2}$ are the transmission frequencies of each n-PAM signal. We suppose $f_{2}>f_{1}$ and $\Delta f=f_{2}-f_{1}<1 / T$ in order to have spectral overlapping between the transmitted signals. The pulse shaping filter is represented by $g(t)$ and we suppose that the pulse spectrum is a square root raised cosine with roll-off equal to zero.

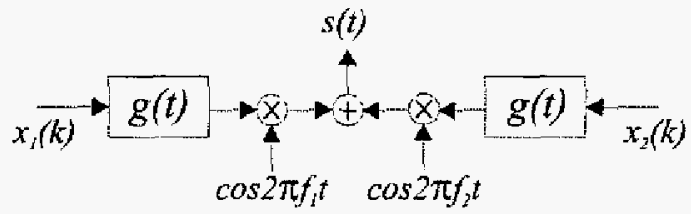

Fig. 1- Transmitter block diagram.

The receiver block diagram is shown in Fig. 2. We assume perfect synchronization at receiver end. The task of low-pass filters (LPF) at mixer outputs is to eliminate the signal around $2 f_{1}, 2 f_{2}$ and $f_{1}+f_{2}$. The filters $g(t)$ are matched filters identical to the transmitter filters ones. The channel is assumed AWGN with bandwidth $B=1 / T$. The noise $n(t)$ at the receiver input is supposed to be white Gaussian with power spectral density $N_{0} / 2$ and mean zero.

The demodulated and sampled signals $d_{1}(m)$ and $d_{2}(m)$, as indicated in Fig. 2, are the inputs of a symbol detection system that delivers the detected signals $\hat{x}_{1}(m)$ and $\hat{x}_{2}(m)$ 


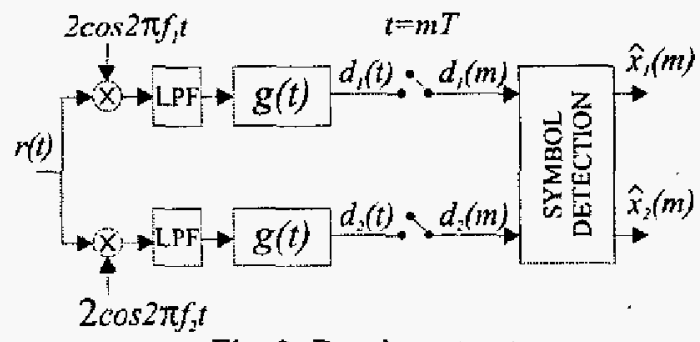

Fig. 2- Receiver structure.

The signal $r(t)$ at the receiver input is given by:

$$
\begin{aligned}
r(t)= & {\left[\sum_{k=0}^{\infty} x_{1}(k) g(t-k T)\right] \cos 2 \pi f_{1} t } \\
& +\left[\sum_{k=0}^{\infty} x_{2}(k) g(t-k T)\right] \cos 2 \pi f_{2} t+n(t) .
\end{aligned}
$$

The signals $d_{1}(t)$ and $d_{2}(t)$ at the output of matched filter are:

$$
\begin{aligned}
d_{1}(t)= & \sum_{k=0}^{\infty} x_{1}(k) g(t-k T) * g(t)+ \\
& \left\{\cos 2 \pi \Delta f t\left[\sum_{k=1}^{\infty} x_{2}(k) g(t-k T)\right]\right\} * g(t)+n_{1}(t), \\
d_{2}(t)= & \sum_{k=0}^{\infty} x_{2}(k) g(t-k T) * g(t)+ \\
& \left\{\cos 2 \pi \Delta f t\left[\sum_{k=0}^{\infty} x_{1}(k) g(t-k T)\right]\right\} * g(t)+n_{2}(t) .
\end{aligned}
$$

where * indicates convolution and the noise terms $n_{1}(t)$ and $n_{2}(t)$ can be written as

$$
\begin{aligned}
& n_{1}(t)=\left[2 n(t) \cos 2 \pi f_{1} t\right] * g(t) \\
& n_{2}(t)=\left[2 n(t) \cos 2 \pi f_{2} t\right] * g(t)
\end{aligned}
$$

Since $g(t)$ is known and the power spectral density is $N_{0} / 2$, from Eq. (4) and (5), we get the following results to noise terms variance $[4]$ :

$$
E\left\{\left[n_{1}(t)\right]^{3}\right\}=E\left\{\left[n_{2}(t)\right]^{2}\right\}=N_{0}
$$

In this work, it was considered that there is no correlation between $n_{3}(t)$ and $n_{2}(t)$ meaning the worse case condition.

Applying Fourier transform in (2) and (3) and doing some mathematical manipulations we get the expressions for discrete time signals $d_{1}(m)$ and $d_{2}(m)$ :

$$
\begin{gathered}
d_{1}(m)=x_{1}(m)+(1-T \Delta f) \sum_{k=0}^{m} \operatorname{sinc}[(1-T \Delta f)(m-k)] \\
\cdot \cos [\pi T \Delta f(m+k)] x_{2}(k)+n_{1}(m) \\
d_{2}(m)=x_{2}(m)+(1-T \Delta f) \sum_{k=0}^{\infty} \operatorname{sinc}[(1-T \Delta f)(m-k)] . \\
\cdot \cos [\pi T \Delta f(m+k)] x_{1}(k)+n_{2}(m)
\end{gathered}
$$

Knowing that function $\operatorname{sinc}[(1-\Delta f T)(m-k)]$ has a maximum at $m=k$ and decreases when $|m-k|$ increases, we can consider as significant terms in summation in Eq. (7) and (8) only $2 L+1$ samples centered in the instant $m$ where $L$ is a given integer. The original summations with infinite terms $k=0, \cdots, m, \cdots, \infty$ is replaced by the additions of $2 L+1$ terms with $k=0, \cdots, m, \cdots, m+L$. For example, if $L=5$ is used in the case where $T \Delta f=1 / 3$, the powet reduction of summation is less than $0.2 \mathrm{~dB}$.

Supposing causal filters, and changing the reference time in such a way that the most advanced received symbol inside the demodulated signals is at the present instant $\mathrm{m}$, we can rewrite the expressions for $d_{1}(m)$ and $d_{2}(m)$ in the following form:

$$
\begin{aligned}
& d_{1}(m)=x_{1}(m-L)+(1-T \Delta f)\{ \\
& \left.\sum_{j=0}^{2 L} \operatorname{sinc}[(1-T \Delta f)(j-L)] \cos [\pi T \Delta f(2 m+L-j)]\right\} \\
& x_{2}(m-j)+n_{1}(m-L), \\
& d_{2}(m)=x_{2}(m-L)+(1-T \Delta f)\{ \\
& \left.\sum_{j=0}^{2 l} \operatorname{sinc}[(1-T \Delta f)(j-L)] \cos [\pi T \Delta f(2 m+L-j)]\right\} \\
& x_{1}(m-j)+n_{2}(m-L),
\end{aligned}
$$

Notice that the demodulated signals contain the desired symbol plus Gaussian noise and several $(2 L+1)$ interference terms originated from another user. In fact, the interfering signal is equivalent to the output of a linear time variant FIR filter that has the second user symbol as its input.

The Figure 3 shows an equivalent model that represents the system since the transmission up to the demodulation.

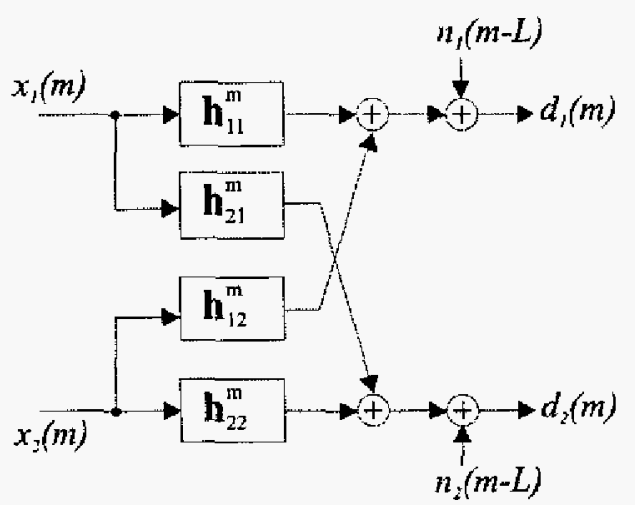

Fig.3- Equivalent Model.

The linear filters $h_{11}^{m}, h_{21}^{m}, h_{12}^{m}$ and $h_{22}^{m}$ can be written in vectorial form as follows:

$$
\mathbf{h}_{11}^{\mathbf{m}}=\mathbf{h}_{22}^{\mathrm{rn}}=\left[\begin{array}{lllllll}
0 & 0 & \cdots & 1 & \cdots & 0 & 0
\end{array}\right]
$$




$$
\mathbf{h}_{12}^{\mathrm{m}}=\mathbf{h}_{21}^{\mathrm{m}}=\left[\begin{array}{lllll}
h_{0}(m) & \cdots & h_{j}(m) & \cdots & h_{2 L}(m)
\end{array}\right]
$$

where

$h_{j}(m)=(1-T \Delta f) \operatorname{sinc}[(1-T \Delta f)(j-L)] \cos \pi T \Delta f(2 m+L-j)$ and $j=0, \cdots, 2 L$.

The problem after this point is how to detect the transmitted sequences $\left\{x_{1}\right\}$ and $\left\{x_{2}\right\}$ from the demodulated signals $d_{1}(m)$ and $d_{2}(m)$. This is the issue of next section.

\section{SYMBOL DETECTION}

If the symbol detection is performed directly from demodulated signals $d_{1}(m)$ and $d_{2}(m)$, without any interference cancellation, the symbol etror rate would be very high, as shown by simulation results presented in next section. Then, it is necessary to introduce some interference cancellation in the receiver scheme. Notice although the filter $\mathbf{h}_{11}^{n h}, \mathbf{h}_{n}^{m}, \mathbf{h}_{12}^{\text {gh }}$ and $\mathbf{h}_{21}^{m}$ in the model are time variant filters, they are known and are given by Eq. (11) and (12). In this work we consider two schemes where interference cancellation is performed first than signal detection. We describe these schemes in the sequel.

\subsection{Detection scheme}

It is evident that each demodulated signal can be thought like a mixture between a desired signal from a given source and a second interfering signal from another source convolved with a linear time variant FIR(TVFIR) filter. Source separation for a very similar situation, except the assumption the filters are time invariant, is discussed in the literature [5]-[6].

If we process the demodulated signals $d_{1}(m)$ and $d_{2}(m)$ with the two input and two output scheme illustrated by Figure 4 , it is straightforward verifying that we have the signals separation when the following conditions are satisfied:

$$
\begin{gathered}
\mathbf{f}_{11}^{\mathrm{m}}=\mathbf{f}_{22}^{\mathrm{n}}=\mathbf{h}_{11}^{\mathrm{m}}=\mathbf{h}_{22}^{\mathrm{m}}, \\
\mathbf{f}_{12}^{\mathrm{m}}=\mathbf{f}_{21}^{\mathrm{m}}=\mathbf{h}_{12}^{\mathrm{n}-1}=\mathbf{h}_{21}^{\mathrm{m}-1} .
\end{gathered}
$$

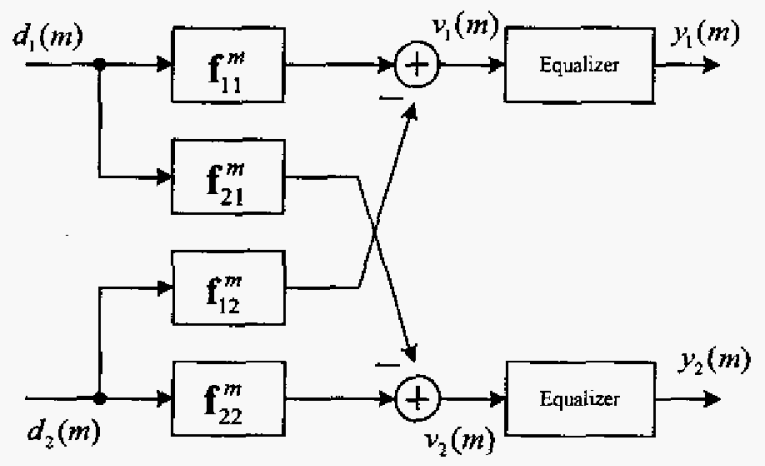

Fig.4- Source separation scheme.

Supposing $L=1$, with no loss of generality, and using the Eq. (13)-(14), the separated signals $v_{l}(m)$ and $v_{2}(m)$ are:

$$
\begin{aligned}
v_{1}(m) & =c_{0}(m) x_{1}(m)+c_{1}(m) x_{1}(m-1)+ \\
& +c_{2}(m) x_{1}(m-2)+c_{3}(m) x_{1}(m-3)+ \\
& +c_{4}(m) x_{1}(m-4)+n_{u_{1}}(m) \\
v_{2}(m) & =c_{0}(m) x_{2}(m)+c_{1}(m) x_{2}(m-1)+ \\
& +c_{2}(m) x_{2}(m-2)+c_{3}(m) x_{2}(m-3)+ \\
& +c_{4}(m) x_{2}(m-4)+n_{u_{1}}(m)
\end{aligned}
$$

where

$$
\begin{gathered}
c_{0}(m)=-h_{0}(m-1) h_{0}(m), \\
c_{1}(m)=-h_{0}(m-1) h_{1}(m)-h_{1}(m-1) h_{0}(m-1), \\
c_{2}(m)=1-\left[h_{0}(m-1) h_{2}(m)+h_{1}^{2}(m-1)\right. \\
\left.+h_{2}(m-1) h_{0}(m-1)\right] \\
c_{3}(m)=-h_{1}(m-1) h_{2}(m)-h_{2}(m-1) h_{1}(m-2), \\
c_{4}(m)=-h_{2}(m-1) h_{2}(m-2), \\
n_{u_{1}}(m)=n_{1}(m-2)-h_{0}(m-1) n_{2}(m-1) \\
-h_{1}(m-1) n_{2}(m-2)-h_{2}(m-1) n_{2}(m-3) \\
n_{v_{2}}(m)=n_{2}(m-2)-h_{0}(m-1) n_{1}(m-1) \\
-h_{1}(m-1) n_{1}(m-2)-h_{2}(m-1) n_{1}(m-3)
\end{gathered}
$$

Notice by Eqs. (15) and (16), that the separated signals are not suitable yet to detection. This is due the fact that symbols present in the signals are dispersed in time, resulting in intersymbol interference (ISI). It worth noting that the coefficients multiplying the transmitted symbols are time dependent. This situation is similar to the transmission of symbol sequences through a linear time variant filter which coefficients are represented

$\mathbf{c}(m)=\left[\begin{array}{lllll}c_{0}(m) & c_{1}(m) & c_{2}(m) & c_{3}(m) & c_{4}(m)\end{array}\right] . \quad$ This implies that we need to equalize $v_{1}(m)$ and $v_{2}(m)$ before detection.

The equalization can be performed using a linear time variant FIR filter. The equalizer tap weight coefticients $\left\{w_{i}(m)\right\}$ for a given instant $m$, as shown by Fig. 4 , may be given as the solution that minimize the mean square value of the error

$$
e_{i}(n)=x_{i}(m-D)-y_{i}(m), \quad i=1,2,
$$

where $x_{i}(m-D)$ is the desired symbol, $\mathbf{D}$ is a given delay and $y_{i}(m)$ is the output of equalizer. Actuatly, the above defined criterion, results in a Wiener filtering for each instant $m$.

For $v_{1}(m)$ equalization, the tap weight coefficients of equalizer, represented by vector $\mathbf{w}_{1}(m)$, for a given instant $m$ should be: 


$$
\mathbf{w}_{1}(m)=E\left\{\mathbf{v}_{1}(m) \mathbf{v}_{\mathrm{j}}^{T}(m)\right\}^{-1} E\left\{\mathbf{v}_{1}(m) x_{i}(m-D)\right\}
$$

where $\mathbf{v}_{1}(m)=\left[\begin{array}{lll}v_{1}(m) & \cdots & v_{1}(m-N)\end{array}\right]^{T}$ and $N$ is the number of coefficients of the equalizer.

DFE equalizer with time variant forward and feedback filters obviously could be also used to perform the equalization. The MSE criterion should be used to find the filters coefficients for each instant $m$. In this case, for $\mathrm{v}_{1}(\mathrm{~m})$ signal equalization, the forward filter coefficients $\mathbf{f}_{w}(m)$ is given by the equation:

$$
\mathbf{f}_{\mathbf{w}_{1}}(m)=E\left\{\mathbf{v}_{1}(m) \mathbf{v}_{1}^{T}(m)\right\}^{-1} E\left\{\mathbf{v}_{1}(m) x_{1}(m-D)\right\}
$$

where $\mathbf{v}_{1}(m)=\left[\begin{array}{lll}v_{1}(m) & \cdots & v_{1}(m-D)\end{array}\right]^{T}, \quad x_{1}(m-D) \mathbf{x}_{1}(m-$ D) is the desired symbol, and $D$ is the decision delay. The feedback filter, represented by $f_{b}(m)$, is given by:

$$
f_{b_{i}}=\sum_{i=0}^{D} f_{u i}(m) c_{D+i-i}(m-i), l=1, \cdots, M
$$

where $c_{r}(m)$ are given by Eqs. (17)-(21), $M$ is the number of coefficients in the feedback filter and $f_{w_{1}}(m)$ are the coefficients of the feedforward filter.

Then, after equalization the signals from both frequencies (users) can be detected. However, another strategy may be used as described in next section.

\subsection{Alternative Approach}

A DFE-like equalizer with time variant feedforward and feedback filters could be also used to perform the detection, as depicted in Fig. 5, since we are dealing with an interference removal issue.

From Eq. (9) and (10), and assuming $L=1$, we can verify that the scheme represented in Fig. 5 also detects the symbols. Symbols originated by an user in demodulated signal are cancelled with the feedback of delayed decision. After that the time disperse signal from another user that remains is equalized by a TVFIR filter $\left(f_{1}^{m}\right.$ or $f_{2}^{m}$ ) before the decision. Notice the linear equalizers $f_{1}^{m}$ and $f_{2}^{m}$ could be replaced also by a DFE equalizer with time variant filter.

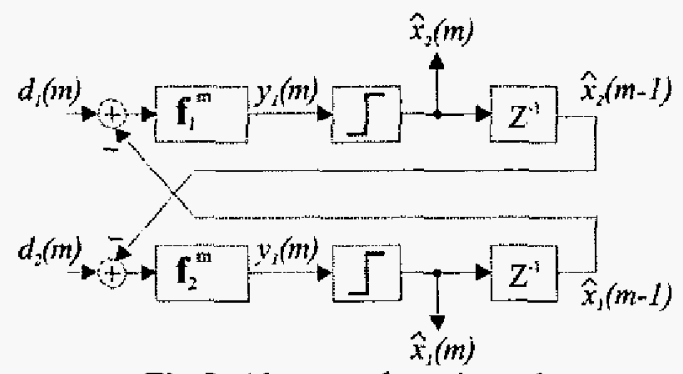

Fig.5- Alternate detection scheme.

Ideally, we cotald obtain the separated signal $y_{1}(m)$ and $y_{2}(m)$ :

$$
\begin{aligned}
& y_{1}(m)=x_{2}(m) h_{0}(m)+n_{1}(m-1), \\
& y_{2}(m)=x_{1}(m) h_{0}(m)+n_{2}(m-1) .
\end{aligned}
$$

Depending on the value of $h_{0}(m)$ it should bave a noise amplification process and the system performance should be poor. It should be necessary to spend more power in the transmission to keep the performance or use strategies to reduce only the noise power as described in [7]. Applications of such strategies in this problem are still being investigated and were not implemented in this work.

We have verified during simulations, that this scheme works satisfactorily only in conditions of high signal-to-noise ratio (SNR). Despite its poor performance in low SNR environments, it remains interesting due to its simpler and flexible structure compared with the first one making it more suitable for adaptive processing.

Furthermore, the processing with the altemative approach leads to the preservation of an AWGN channel with an attenuation of the original signal.

\section{SIMULATION RESULTS}

We have simulated a system with binary PAM signals, $\Delta f T=1 / 3$, and $L=1$. In this case, the filters for the equivalent model described in Fig. 3 ate:

$$
\begin{aligned}
& \mathbf{h}_{11}^{m}=\mathbf{h}_{22}^{m}=\left[\begin{array}{lll}
0 & 1 & 0
\end{array}\right], \\
& \mathbf{h}_{12}^{m}=\mathbf{h}_{21}^{m}=\left[\begin{array}{lll}
h_{0}(m) & h_{1}(m) & h_{2}(m)
\end{array}\right], \\
& \text { where } \\
& h_{0}(m)=0.2757 \cos [\pi(2 m+1) / 3] \text {, } \\
& h_{1}(m)=0.6667 \cos [\pi(2 m) / 3] \\
& h_{0}(m)=0.2757 \cos [\pi(2 m-1) / 3] \text {. }
\end{aligned}
$$

It worth noting that the filter coefficients $h_{0}(m), h_{1}(m)$, and $h_{2}(m)$ although time variant, are periodic with period $P=3$, resulting in only three possible filters. This fact simplifies the implementation of the detection schemes.

Fig. 6 shows the symbol error rate (SER) at different values of symbol energy by noise density $\left(E_{b} / N_{0}\right)$ for the proposed detection scheme and the altemative one, compared with the situation when the detection is done directly from demodulated signal without any interference cancellation. For the first detection scheme, the used equalizer was a DFE equalizer with a decision delay equals 2 , a time variant feedforward filter with 3 taps and a time variant feedback filter with 4 taps optimized by the MSE criterion. The equalizer of the altemative scheme is a DFE one with a decision delay equals zero, feedforward filter with 1 tap and feedback filter with 2 taps also optimized by the MSE criterion. 


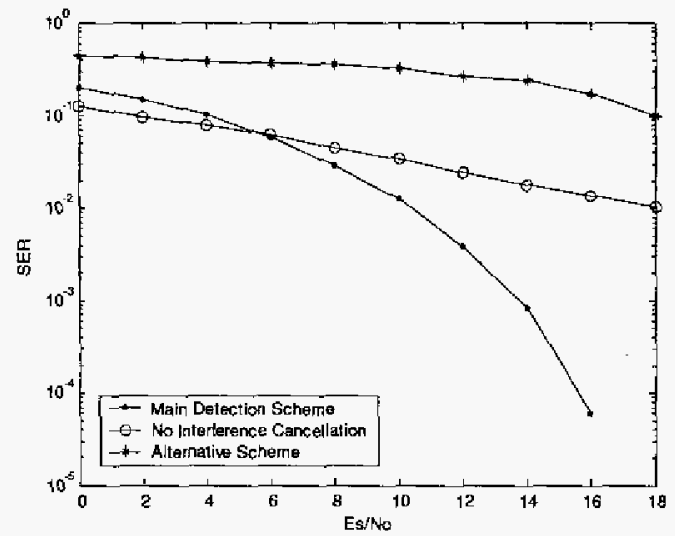

Fig.6- Symbol error rate

The superior performance of the first scheme is evident. The reason for the difference in performance is the cancellation and equalization process in each scheme.

In altenative approach, there is a cross-feedback between users signals in order to make the principal user cancellation and to preserve the terms of interfering user. Then, a DFE equalizer with a decision delay equal to zero, eliminates the delayed symbols and maintains the symbols at instant $\mathrm{m}$ that are multiplied by the coefficient $h_{0}(m)$, Eqs. (28) and (29), which has low amplitude, resulting in big attenuation. Further, this process amplifies the Gaussian noise and makes the ratio of the signal to the noise tighter than for the first scheme, where the best symbol can be chosen.

To cope with this problem, we are studying the impact on the orthogonality when normalizing the channel to a more favorable situation.

\section{CONCLUSIONS AND PERSPECTIVES}

We have proposed a mathematical modeling for systems transmitting non-orthogonal signals with spectral overlapping.

Using the fact that, from the mathematical model, the system can be viewed as a source separation problem, we proposed an interference cancellation scheme for detection of the involved signals.

Simulations have shown that preliminary results from the proposed technique to detect two non-orthogonal n-PAM signals with spectral overlapping for a band-limited channel increases the performance of the system in terms of symbol error tate. The advantage of presented technique (main detection scheme) is low complexity invoived even considering the time variant system.

A direct extension of this wotk is the complex modulation. A generalization of the Viterbi algorithm for multi-user and time variant systems is another technique that is being investigated to be applied in the problem of spectral overlapping.

Further, the consideration of channel estimation is another research line to be tracked, since so far we have assumed perfect channel estimation.

\section{REFERENCES}

[1] S. B. Weinstein and P. M. Ebert, "Data transmission by frequency division multiplexing using the discrete Fourier transform", IEEE Trans. Commun. Technol. Vol. COM-19, no. 5 , pp. 628-634, Oct.1971.

[2] R. L. Pickholtz, D. L. Schilling, L. B. Milstein, "Theory of Spread-Spectrum Communications-A Tutorial". IEEE Trans. On Commun. Vol. COM-30, No. 5, May 1982.

[3] E. C. Giraudo, F. R. Baldini and R. R. Scarabucci, "On the \{m-QAM $\}^{2}$ Modulation". IEEE Commumications Letters, Vol. 5 , $\mathrm{n}^{\circ}, 10$, pp. 426-428, Oct. 2001.

[4] Papoulis, A., Probability, Random Variables, and Stocachastic Process, McGraw-Hill, International Student Edition, 1965.

[5] D. Yellin, and E. Weinstein, "Multichannel Signal Separation: Method and Analysis", IEEE Trans. on Signal Processing. Vol. 44, $\mathrm{n}^{\circ} 1$, Jan. 1996.

[6] E. Weinstein. M. Feder, and A. V. Oppenheim, "Multichannel Signal Separation by Decorrelation", IEEE Trans. on Speech and Audio Proc. Vol. 1, n 4, Oct. 1993.

[7] J. F. Galdino, E.L. Pinto and M. S. Alencar, "Using Denoising at the Receiver Front-end for Frequency-Selective Channels", IEEE Trans. On Commu. , Vol. 51, $1^{\circ}$ 5, pp.727-729, May 2003. 